\title{
A Study of Degradation of Historic Parchment Using Small-Angle X- Ray Scattering, Synchrotron-IR, and Multivariate Data Analysis
}

\author{
Alenka Možir ${ }^{1}$, Lee Gonzalez ${ }^{2}$, Irena Kralj Cigić ${ }^{1}$, Tim J. Wess ${ }^{2}$, Ira Rabin ${ }^{3}$, Oliver Hahn ${ }^{3}$, \\ Matija Strlič ${ }^{* *}$ \\ ${ }^{1}$ University of Ljubljana, Faculty of Chemistry and Chemical Technology, Aškerčeva 5, 1000 \\ Ljubljana, Slovenia \\ ${ }^{2}$ School of Optometry and Vision Sciences, Maindy Road, Cardiff University, Cardiff CF24 4LU, \\ United Kingdom \\ ${ }^{3}$ Federal Institute for Materials Research and Testing, 12200 Berlin, Germany \\ ${ }^{4}$ University College of London, Centre for Sustainable Heritage, The Bartlett School of \\ Graduate Studies, 14 Upper Woburn Place, London, WC1H ONN, United Kingdom
}

*corresponding author. E-mail: m.strlic@ucl.ac.uk, Tel.: +44 207679 5994, Fax: +44 2079161887

\begin{abstract}
Parchment has been in use for thousands of years, and has been used as the writing or drawing support for many important historic works. A variety of analytical techniques are currently used for routine assessment of the degree of denaturation of historic parchment; however, because parchment has a heterogeneous nature, analytical methods with high spatial resolution are desirable.

In this work, the use of small-angle X-ray scattering (SAXS) and synchrotron-IR (SR-IR) was examined in conjunction with multivariate data analysis, to study degradation of an extended set of historic parchment samples and particularly to investigate the effect of lipids and the presence of iron gall ink on the degradation processes. In the data analysis, shrinkage temperature, lipid content, sample age, presence of ink, and accelerated degradation were included. The analysis of loading factors in partial least squares regression and principal component analyses based on SAXS, SR-IR, and other analytical and descriptive data reveals the effect of lipid removal on diffraction patterns and lipids are found to cause the degradation process in parchment to accelerate. The effect of iron gall ink is also evident, although the mechanism of ageing is different to that of natural ageing in the absence of ink. In addition, an historic parchment score from ca. 1750 is examined demonstrating the significant effect of iron gall ink, and lipids and inorganic soiling on its increased degradation.
\end{abstract}

\section{Keywords}

Small angle X-ray scattering; Multivariate data analysis; Degradation; Collagen; Lipids; Iron gall ink

\section{Introduction}

Parchment has been used as a writing medium for more than two millennia [1]. Large parchment-based collections of books and documents are kept in heritage institutions [2], and it is important to understand and manage material change leading to degradation of material properties, either induced by the environment or by material properties, including the properties of writing and drawing media. Of these, iron gall inks, composed mainly of gallotannins, vitriol (iron(II) sulfate), Arabic gum and water, are particularly known for their corrosive properties [3]. 
Parchment and its main component collagen [4] are complex materials due to their natural heterogeneity, additionally exacerbated by natural degradation. The collagen molecule consists of three polypeptide chains with a known amino acid sequence forming a triple helix [4]. This unique structure of collagen, with numerous intermolecular bonds, allows for excellent mechanical properties and chemical stability of a parchment object. Due to numerous degradation processes the collagen structure may become unfolded, turning into disordered gelatine, which can reduce a parchment document's ability to be fit for purpose [5]. It has been shown that lipids in parchment may significantly promote this process, and that oxidative stress could play a role [6-8], however, more evidence is still needed to confirm the relative importance of lipid-driven degradation processes.

The routinely used analytical methods and techniques, such as thermal analysis and XRD are destructive or micro-destructive [6,9], and benchtop spectroscopic methods such as FTIR and FT-Raman [10-12] may not provide information that is sufficiently spatially resolved. The development of techniques that would satisfy the need for higher spatial resolution is still called for.

Shrinkage temperature is often used as an indicator of hydrothermal stability because it is directly connected with the molecular structure of collagen and the level of deterioration. It is also well-understood by the end-user community and used in condition assessment of parchment objects [13-16].

To extract chemical information, infrared (IR) spectroscopy is one of the most popular spectroscopic tools for the study of parchment $[17,18]$. The amide I vibration represents the dominant peak in a parchment spectrum, occurring at $1600-1700 \mathrm{~cm}^{-1}$ and represents a series of stretching vibrations of the $\mathrm{C}=\mathrm{O}$ and $\mathrm{C}-\mathrm{N}$ functions. The amide II peak occurs at $1510-1580 \mathrm{~cm}^{-1}$, is more complex than amide I as it contains a series of bands, and depends predominantly on the $\mathrm{N}-\mathrm{H}$ bending vibrations. During degradation, the position and the intensity of the absorbance maxima of the amide I and II bands changes and can be used to characterise collagen conformational changes. An increase of $\mathrm{OH}$ stretching can be noted at wavenumbers 3400 and $1650 \mathrm{~cm}^{-1}$, leading to an increase in the intensity of the amide I peak $\left(1650 \mathrm{~cm}^{-1}\right)$. Carbonyl compounds are observed as a shoulder on the amide I and may also cause an increase of the peak area [19].

Orientation [5], long-range order [13,16] and diameter [20] of collagen fibrils and their intramolecular or intermolecular interactions $[20,21]$ can be determined using X-ray diffraction (XRD). These structural parameters are concomitant with the condition of a parchment object [13]. XRD has been used to study changes in collagen structure and accumulation of disordered gelatine during ageing $[5,13]$. This is a relevant indicator for the level of degradation of a historic parchment object [5].

The rapid development of synchrotron-based analytical instrumentation allows for the use of micro XRD [5] and small-angle X-ray diffraction (SAXS) [22], which enable point-to-point microscale mapping of structural features of sizes ranging from 1-100 $\mathrm{nm}[16,20]$. Synchrotron-based diffraction techniques are often favoured over standard ones because of the ultra-bright light, more sensitive detectors and better attainable spatial resolution $[13,23]$. This is of importance in studies of heritage objects, as the sample size needs to be as limited as possible.

In this work we investigate the use of synchrotron methods, both SAXS and IR, in conjunction with multivariate data analysis, to study parchment degradation and the effect 
of the lipid fraction and iron gall ink on the degradation processes. Additionally, shrinkage temperature was determined for all the samples, while the content of lipids was determined gravimetrically, after extraction. This combination of traditional and micro-analytical methods allowed us to examine the effects of material composition on localised degradation of real (sacrificial) historic parchment samples.

\section{Material and Methods}

\section{Samples}

In the study, 9 historic parchment documents of variable origin were used, obtained commercially or through donations, produced between 1480 AD and 1900 AD. The samples were sacrificial, meaning that they were of no documentary or historical value and could be used for scientific research. An additional document (score) from 1750 AD, exhibiting localised degradation, was used.

Lipids were extracted from samples with hexane (HPLC grade, J.T.Baker, USA) using Soxhlet extraction, the duration of extraction was $0 \mathrm{~h}$ (immersion only), $1 \mathrm{~h}$ or $4 \mathrm{~h}$. After extraction, the samples were dried in vacuum and equilibrated at room temperature and relative humidity. The amount of extracted (hexane-soluble) material was determined gravimetrically.

One half of all parchment samples (extracted and non-extracted) were then subjected to accelerated degradation in a climatic chamber (Vötsch Climatic chamber, Type VC 0020) at $80{ }^{\circ} \mathrm{C}$ and $65 \% \mathrm{RH}$ for 18 days. The other half was not subjected to degradation. Thus we obtained a set of 40 samples, differently delipidised and degraded, some additionally containing iron gall ink inscriptions, as verified using iron gall ink test paper (Conservation by Design, Bedford, UK).

\section{Shrinkage temperature}

Two different systems were used for measurement of shrinkage temperature:

(i) The shrinkage temperature of most parchment samples was determined as described in a previous publication [8].

(ii) The shrinkage temperature of the historic score from 1750 AD was determined using a microscope Leica Gallen III (10x) equipped with a hot table. The temperature programme and the determination of shrinkage temperature were the same as above.

After sampling with a scalpel, a few collagen fibres were immersed in distilled water on a microscope slide with a central concavity. After separation, the fibres were covered with a slide and placed on the micro hot table. The temperature was increased at $2{ }^{\circ} \mathrm{C} \mathrm{min}^{-1}$ until the temperature at which shrinking was just observed (this temperature was noted), and then continued until the process completed.

The average standard deviation of shrinkage temperature measurement was $0.6-0.8{ }^{\circ} \mathrm{C}$, and the RSD 1-1.5\%. The average standard deviation and RSD were determined from five independent measurements of a sample $(n=5)$. The amount of sample needed for this analysis is $30-50 \mu \mathrm{g}$. 


\section{Small Angle X-ray scattering}

2D small angle X-ray scattering (SAXS) diffractograms were collected on the non-crystalline diffraction beamline (122) at the Diamond Light Source (Harwell Science and Innovation Campus, UK). The scattering of X-rays by a parchment sample was performed with X-rays of $0.154 \mathrm{~nm}$. The sample to detector distance was $5.5 \mathrm{~m}$. Three measurements were taken at different positions of the same sample.

\section{Synchrotron Infrared Spectroscopy}

To acquire transmission FT-IR spectra, micro samples were prepared in a Diamond micro compression cell and measured at a room temperature with a Continuum microscope (Nicolet) equipped with a liquid nitrogen cooled MCT detector. The measurements were performed using infrared synchrotron radiation from the BESSY II storage ring at the IRIS beamline. A 32x Cassegrain objective was used to focus the beam onto the sample. The area of investigation was $50 \mu \mathrm{m} \times 50 \mu \mathrm{m}$ and a total of 256 scans were collected per sample spectrum in the wavenumber range between 4,000 and $650 \mathrm{~cm}^{-1}$.

All experimental data is available as Electronic Supplementary Material.

\section{Multivariate Data Analysis}

Partial least square regression (PLS) and principal component analysis (PCA) and were performed using Unscrambler v.9.7 (CAMO, Trondheim) and IBM SPSS Statistics 17 (SPSS Inc., Chicago, USA), respectively.

PCA is a mathematical visualisation procedure that transforms a number of correlated variables into a (smaller) number of uncorrelated variables called principal components. The objective of principal component analysis is to reduce the dimensionality (number of variables). PCA analysis is used for grouping of data sets according to their similarities and differences $[24,25]$. For all PCA work in this paper, untreated centred spectra were used. On the other hand, PLS enables spectral and measured/observed information to be correlated. The quality of results in both cases depends on a number of factors, among which the quality of the reference measurements (X-ray diffractograms, IR spectra etc.) is the most important $[24,26]$.

In both PCA and PLS, loading vectors can be understood as the weights for each original variable, and the loading plot will give an overview of the importance of the original variables. In PLS or PCA, in the loadings vs. variables plot, two variables that are both positive or both negative are positively correlated, otherwise they are negatively, i.e. anticorrelated. In PCA, in the PC1 loadings vs. PC2 loadings plot, two variables are correlated if their loadings appear at close to $0^{\circ}$ relative to the origin, if at ca. $180^{\circ}$ they are negatively or anti-correlated, and if at ca. $90^{\circ}$, then the two variables are not correlated.

\section{Results and Discussion}

\subsection{Examination of the effect of lipids with SAXS}

The main character of a collagen molecule is the triple helical structural motif [27]. During ageing, its helical structure transforms into a random gelatinous structure. High proportions of gelatine can make parchment objects become unfit for the purpose of manual handling. Since parchment is structurally and chemically inhomogeneous, methods with high spatial 
resolution are needed [13] and synchrotron (SR)-based analytical methods are ideally suited for its characterization as they are often non-destructive or require a microscopic sample, often a single fibre [23]. X-ray analysis had already proven useful for the characterisation of collagen [16,20-22].

In this research, 9 sacrificial historic samples were used, originating between 1480 AD and 1900 AD. Shrinkage temperature was determined using thermal microscopy, and small angle $X$-ray (SAXS) diffractograms were collected for the whole sample set. The Bragg order reflection that corresponds to the lipid phase of parchment samples appears at approximately $0.22 \mathrm{~nm}^{-1}$ [6]. After $1 \mathrm{~h}$ of extraction the $0.22 \mathrm{~nm}^{-1}$ reflection is still observed (Figure 1). However, after $4 \mathrm{~h}$ of extraction the $0.22 \mathrm{~nm}^{-1}$ reflection no longer appears indicating that the lipid phase of the parchment sample has been removed (Figure 2).

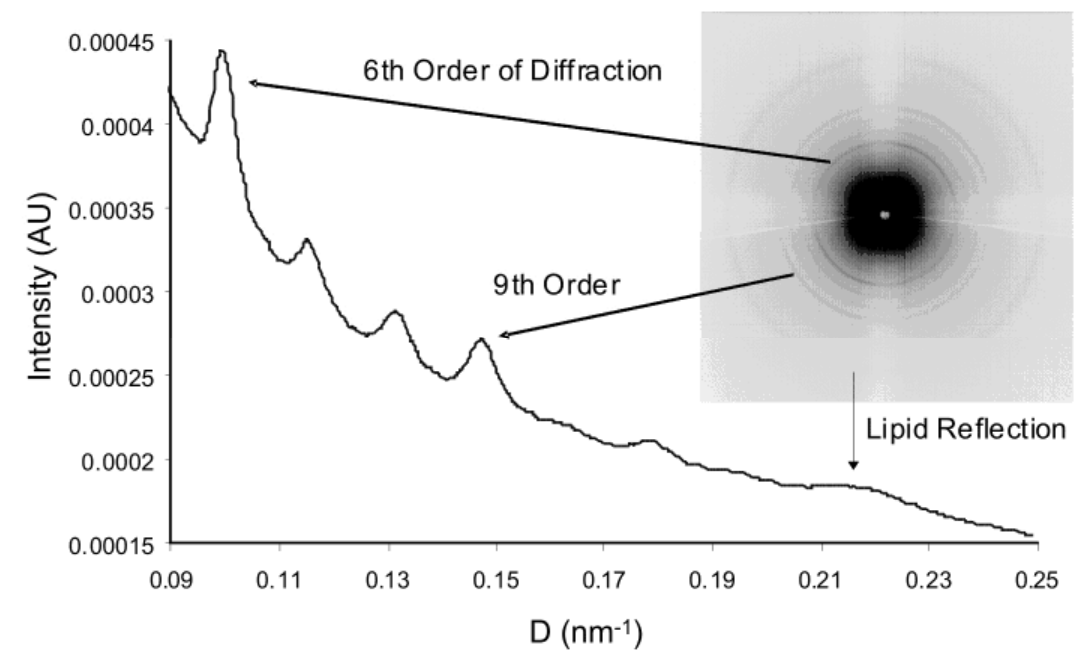

Figure 1: 2D Diffraction image from sample OR10 from 1731, no accelerated degradation, after 1-h extraction and the corresponding linear trace. The reflection from the lipid phase is still apparent.

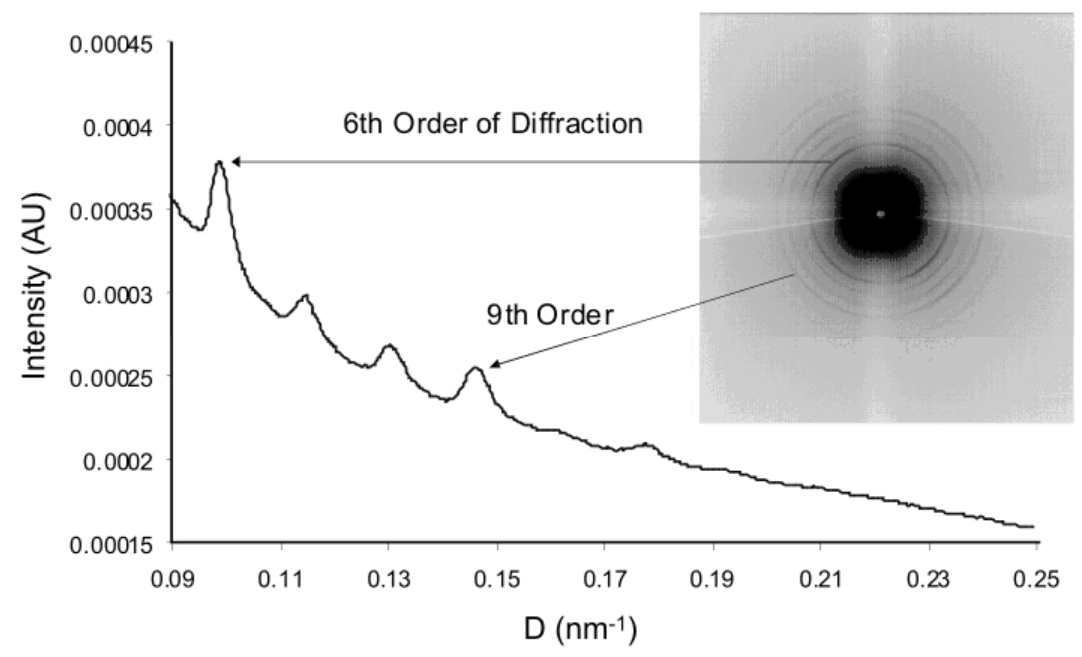

Figure 2: 2D Diffraction image and the corresponding linear trace from sample OR10 from 1731, no accelerated degradation, after 4-h extraction. The reflection corresponding to the lipid phase is no longer visible, indicating that lipids have been removed.

Partial least squares (PLS) regression is particularly useful to examine quantitative relationships between whole spectra and reference analytical information. Based on SAXS integrated intensity profiles for historic parchment samples, a PLS calibration (Table 1) was 
calculated for lipid content of parchment samples, in which the actual lipid content was determined gravimetrically. Using cross-validation, the optimal calibration was obtained using 15 latent variables and no spectral pre-treatment. The calibration itself is not particularly useful for quantitative prediction of lipid content based on diffraction patterns due to the high root mean square error of cross validation and low $R^{2}$. This might be a reflection of the quality of the reference data on lipid content obtained gravimetrically, but also a consequence of the fact that sample sizes of samples used for extraction and for SAXS are significantly different.

However, the calibration does indicate that there is a quantitative correlation and thus it does allow for analysis of the loading plot (Figure 3). From the latter, it can be concluded that while meridian reflections of the 6th-10th order do contribute to the calibration, the lipid reflection at $0.22 \mathrm{~nm}^{-1}$ is much more prominent in the loading plot than in original diffractograms (Figure 1 ) indicating its important contribution to the calibration. The loading plot also indicates that there is an anti-correlation between lipid content and meridian reflections of the $6^{\text {th }}-10^{\text {th }}$ order.

Table 1: Partial least squares (PLS) calibration and cross validation data for determination of lipid content in historic parchment $(n=25)$. RMSE - root mean square error.

\begin{tabular}{ccccc} 
& Slope & Offset & RMSE [\%] & $R^{2}$ \\
\hline Callibration & 0.44 & 0.65 & 0.16 & 0.44 \\
Cross Validation & 0.32 & 0.79 & 1.41 & 0.23 \\
\hline
\end{tabular}

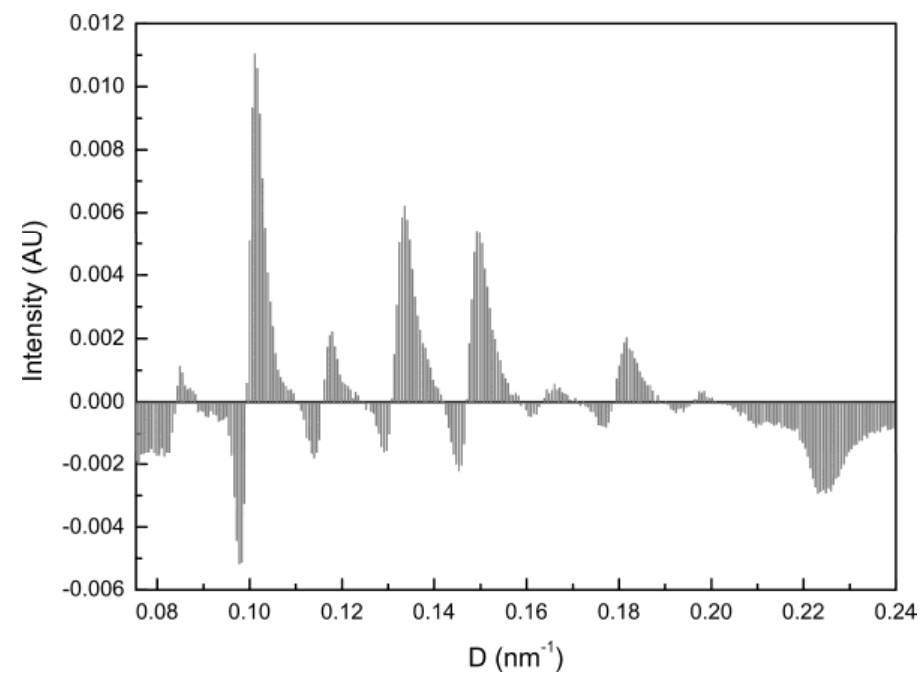

Figure 3: Loading plot of the PLS calibration for prediction of lipid content in historic parchment based on diffractograms $(n=25)$.

The parameters from the diffraction pattern that describe changes in the structural properties of the collagen lattice are:

(i) Shifts in the periodicity of meridional peaks i.e. the distance at which they appear in relation to one another. A change in this parameter indicates that the average distance 
between repeating units (in this instance, the gap and overlap region of the staggered array of collagen molecules in the collagen fibril) has changed.

(ii) The full width at half-maximum (FWHM) of the meridional peaks. The FWHM can be used to describe the distribution of gap and overlap distances between collagen molecules along a collagen fibril. If the variation of the gap and overlap period through a collagen fibril increases then the FWHM will increase for all meridional peaks. Since the variation of the repeating alignment of collagen molecules in the collagen fibril is very small, this indicates a structural difference in parts of the collagen fibril.

(iii) The diffuse scatter (represented by the total area under the meridional peaks after background subtraction) arises from X-rays randomly scattering from non-crystalline structures. If the diffuse scatter increases then a greater proportion of the area exposed to $X$-rays is non-crystalline, indicating that more degraded collagen is present.

(iv) The intensity of the meridional peaks. A greater intensity indicates that more repeating units have diffracted the X-rays at the same angle. When compared against the amount of diffuse scatter this parameter can be used to quantify the amount of collagen present in a sample.

The next step was to test whether there is a degradation pattern exhibited by differently degraded historic sample with different lipid content.

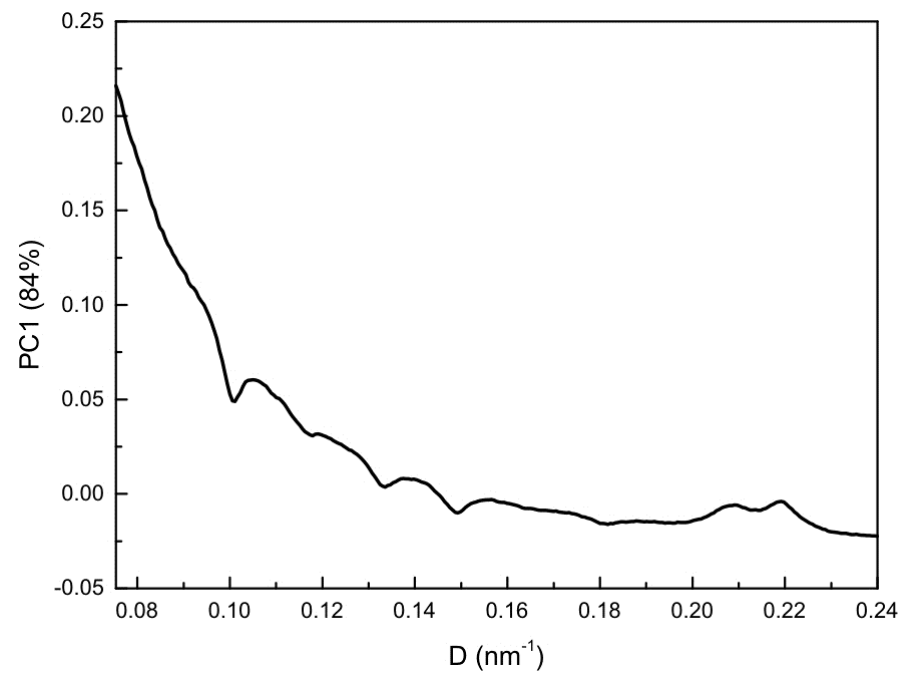

Figure 4: Loading plot of the PCA, using diffractograms of identical historic parchment samples of different age, both additionally degraded and not (but none extracted).

In Figure 4, the loading plot for PC1 accounting for $84 \%$ of the variability is shown, obtained after principal component analysis (PCA) using raw diffractograms of identical historic parchments, pair-wise degraded and non-degraded. None of the samples used in this PCA were extracted with hexane. Since samples of different age were used, both additionally degraded and not, the PCA does not separate samples into clear groups, however, younger and non-degraded samples and older and additionally degraded samples separate along PC1, with older samples tending towards more positive scores.

The loading trace shows that the 6th-9th orders of reflection are weakly anti-correlated with the lipid reflection at $0.22 \mathrm{~nm}^{-1}$, indicating that higher lipid content is associated with a loss of diffraction intensity from the meridional series. Additionally, the diffuse scatter positively 
correlates with lipid reflection, meaning that higher lipid content is also associated with a higher proportion of non-crystalline material in degraded parchment samples. These findings infer that there is more degradation of the collagen fibril with higher lipid concentrations.

In order to make data interpretation clearer, the diffractograms for historic parchment samples were deconvoluted and the data on heights of individual peaks representing reflections of the 6th-10th order was compiled, including the corresponding shrinkage temperatures (ST), i.e. temperatures of denaturation, sample age and whether or not a parchment sample has been subjected to accelerated degradation prior to SAXS measurements.

In Figure 5, correlations between experimental parameters can be explored. The combined variability accounted for by PC1 and PC2 is $77 \%$, leading to separation of samples according to age along PC1 and according to shrinkage temperature along PC2. From the loading plot shown, it is evident that with natural ageing (represented by age in years) the meridional reflections of the 6th-10th order decrease. Age is also anti-correlated with shrinkage temperature (ST), which is commonly understood as a measure of the stability of parchment [13]. The latter is also lower in samples that have been artificially degraded.

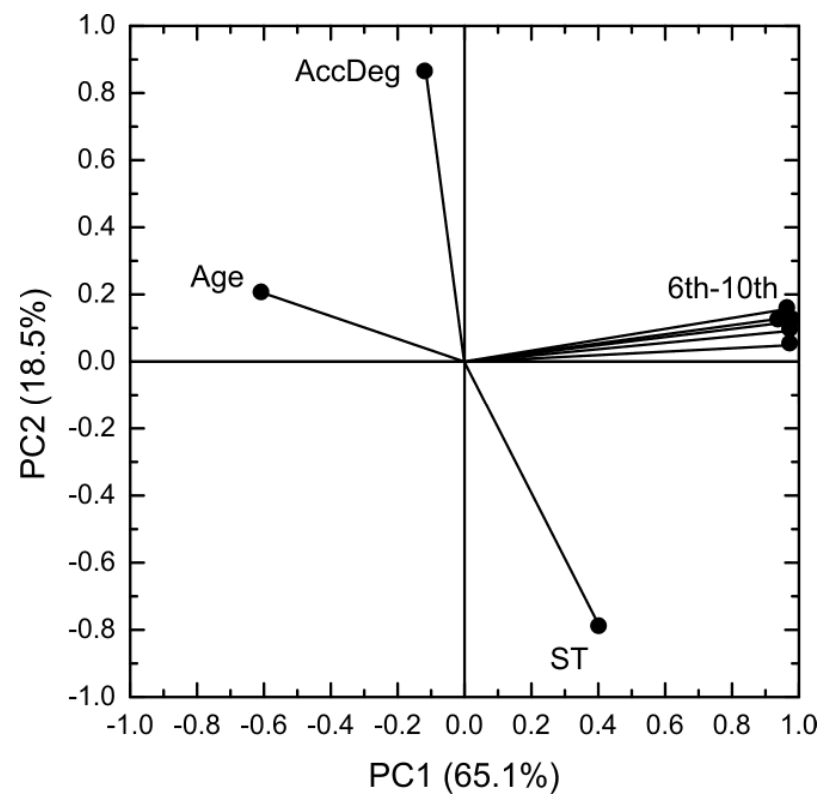

Figure 5: Loading plot for PCA on the basis of data on historic parchment samples: shrinkage temperature (ST), age and whether or not accelerated degradation has been performed (AccDeg) prior to SAXS analysis, represented by peak heights of the $6^{\text {th }}-10^{\text {th }}$ reflection.

\subsection{The effect of iron gall ink studied by SAXS and SR-IR}

Degradation in the presence of inks is an issue where micro-analytical methods are of particular interest. It is known that iron gall ink has highly corrosive properties and rapidly leads to substrate degradation due to the high proportion of acids and transition metals in the ink [3]. It is believed that acid hydrolysis of parchment mediated by iron-gall ink is largely suppressed by calcium carbonate acting as a buffer. It is not known, however, to what extent iron gall ink promotes oxidation of parchment. To investigate this issue, we analysed microsamples from sacrificial parchment samples immediately beneath where ink had been applied. 


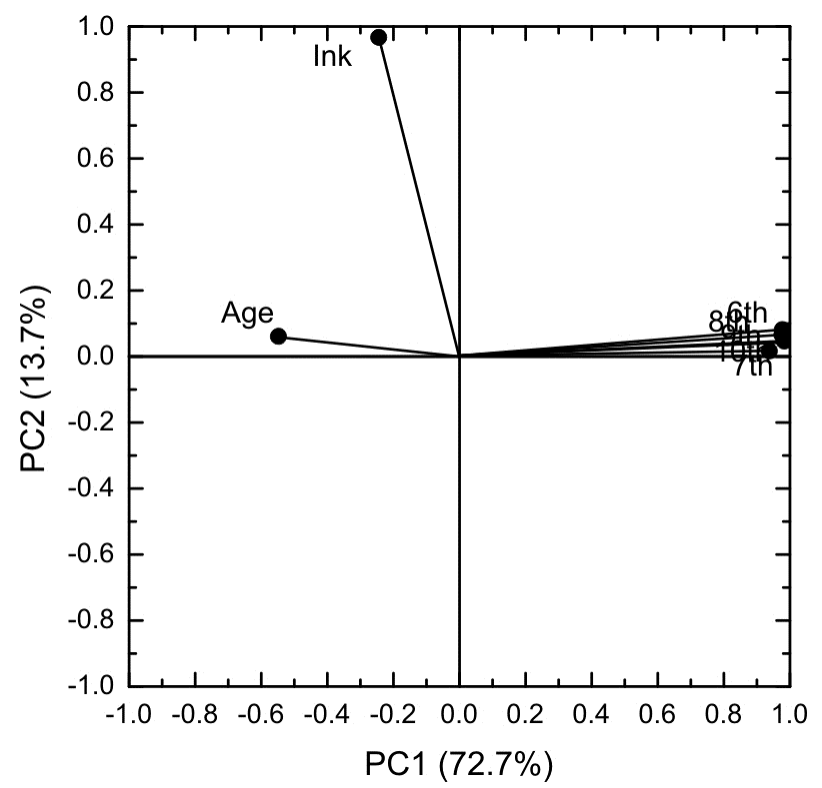

Figure 6: Loading plot for PCA on the basis of data for historic parchment samples: age and whether or not iron gall ink was present at the sampling location. SAXS results are represented by peak heights of the $6^{\text {th }}-10^{\text {th }}$ reflection.

PCA was also carried out with data on historic samples with and without ink, of various age, and of peak heights in SAXS diffractograms. Samples broadly separate according to age (PC1) and according to whether ink was present or not (PC2). An analysis of the loading plot (Figure 6) clearly reveals that sample age anti-correlates with peak height, however, there is little, if any, correlation with the presence of ink. This is of interest, as it may indicate that degradation catalysed by iron gall ink might proceed differently to degradation in its absence.

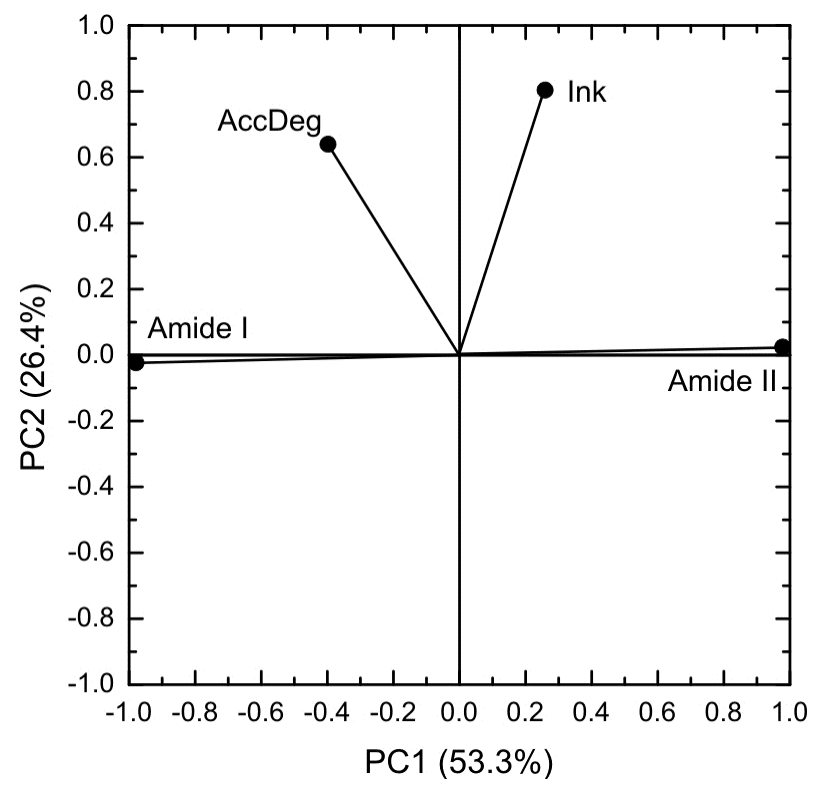

Figure 7: PCA loading plot representing the influence of various experimental factors on relative intensities of amide I and II bands of a parchment from year 1480, differently pre-treated (31 spectra). 
To extract more data, we carried out SR-IR on micro-samples extracted from sacrificial historic samples in the presence and in the absence of iron gall ink. We were particularly interested on how the degradation affects the relative areas of amide I (between 1600 and $1700 \mathrm{~cm}^{-1}$ ) and amide II (between 1510 and $1580 \mathrm{~cm}^{-1}$ ) peaks. Relative peak areas were calculated by deconvolution and normalisation. To visualise the data, PCA was used, and in the scatter plot, samples clearly separate along the PC1 based on the relative amide peak heights, while PC2 separates the samples based on whether they have been exposed to degradation, either due to ink or due to accelerated degradation. The loading plot (Figure 7) represents the influence of various experimental parameters, such as whether accelerated degradation was used prior to measurements (AccDeg) or whether the sample was taken in the immediate vicinity of ink, on the relative intensity of the two amide peaks. It is evident that accelerated degradation leads to a relative decrease of the amide II peak area, while natural degradation in the presence of ink does not have the same effect, again indicating that the mode of degradation is dissimilar.

\subsection{Examination of a historic document with localised damage}

To visualise the issues discussed above, a score in square-note neume notation from 1750 $A D$ was examined. The script was written in iron gall ink and due to use, lipids and soiling accumulated on the corner of the page. Samples were taken at a distance of $1 \mathrm{~cm}$ from each other, starting at the corner and moving in a diagonal line towards the centre of the page. Shrinkage temperature was determined to provide a quick assessment of the extent of degradation. The results are shown in Figure 8. 


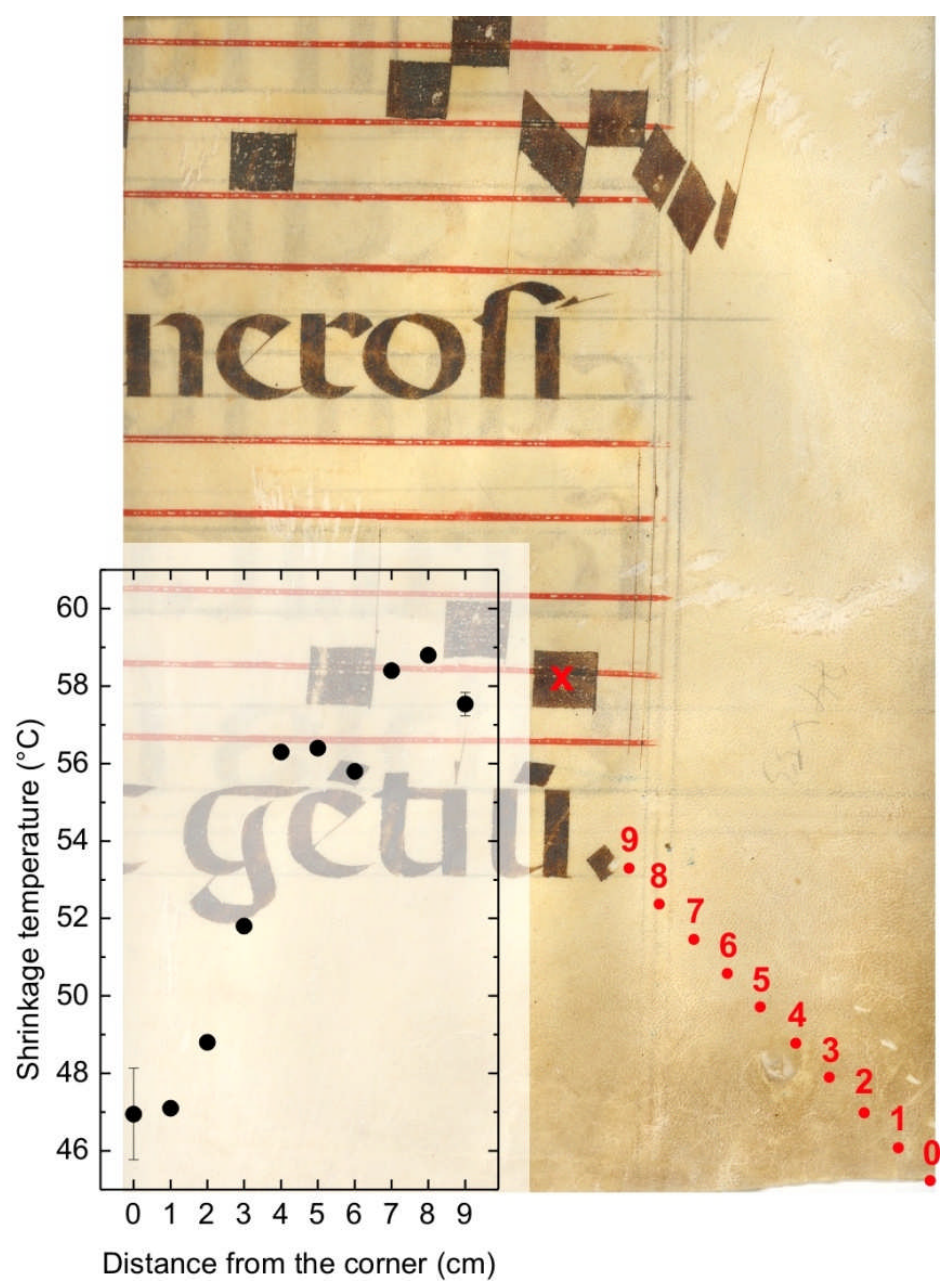

Figure 8: A historic score from $1750 \mathrm{AD}$, sampled at positions 0-9, with shrinkage temperature of the microsamples determined, the results shown in the inset. An additional sample was extracted on the spot marked with ' $x$ ', with iron gall ink application. The error bars represent measurement uncertainty for triplicate analyses.

It is clear that the parchment sheet is more degraded at the corner of the page, where it is reasonable to assume that lipids contributed to degradation. Their presence has amounted to a substantial difference in the shrinkage temperature of $10^{\circ} \mathrm{C}$, as shown in Figure 8 . Additionally, shrinkage temperature was measured beneath iron gall ink spot, marked as ' $x$ ' in Figure 8 , where it was $54.9^{\circ} \mathrm{C}$. This series of results clearly demonstrates that natural degradation of parchment is significantly enhanced by the presence of soiling (incl. added lipids), as a consequence of years of use.

\section{Conclusion}

Degradation of historic parchment was investigated using a set of naturally degraded historic parchments. Additionally, samples were pre-treated using extraction in hexane to remove lipids, and accelerated degradation in a climate chamber. The following can be concluded:

- Using principal component analysis (PCA) and partial least squares (PLS) regression of SAXS data, we have shown that meridian reflections of the 6th-10th order the lipid reflection at $0.22 \mathrm{~nm}^{-1}$ are anti-correlated, indicating that higher lipid content leads to more degradation. We have also shown that the diffuse scatter - from increased disorder - increased with lipid content. 
- PCA was used to examine the correlation between X-ray diffraction peaks, sample age, shrinkage temperature and accelerated degradation. It is evident that with age the intensity of meridional reflections decreases relative to the sample-dependent diffuse scatter, as well as the decrease of shrinkage temperature.

- Examination of the effect of iron gall ink on parchment revealed that samples containing ink degrade differently to naturally aged samples without ink, this conclusion being supported by both the SAXS and SR-IR data.

- Using an original parchment document significantly lower shrinkage temperatures were obtained from samples at the corner of a page, where soiling and lipids, accumulated due to manual handling, has led to more pronounced degradation.

Due to the inhomogeneity of historic parchment, substantial sample sets need to be studied in conjunction with multivariate data analysis, as the effects of factors influencing the degradation of such materials are often difficult to separate and study analytically.

\section{Acknowledgements}

The work was financially supported by the Slovenian Research Agency (programme P10153). We also wish to thank user support of the Diamond Synchrotron, particularly Dr Jen Hiller, Dr Marc Malfois and Professor Nick Terrill of 122 Beamline. We also wish to thank Dr. Ulrich Schade of the IRIS Beamline at the BESSY Synchrotron, and Emanuel Kindzorra and Roman Schütz for their help with sample preparation and measurements.

\section{References}

1 Ryder ML (1964) Parchment - Its history, manufacture and composition. J Soc Archiv 2: 391-399

2 Budrugeac P, Miu L, Bocu V, Wortman FJ, Popescu C (2003) Thermal degradation of collagen-based materials that are supports of cultural and historical objects. J Therm Anal Calorim 72:1057-1064

3 Kolar J, Strlič M (2006) Iron gall inks: on manufacture, characterization, degradation and stabilization. National and University Library, Ljubljana

4 Bailey AJ, Paul RG (1998) Collagen: a not so simple protein. J Soc Leath Technol Chem 82:104-110

5 Kennedy CJ, Hiller JC, Lammie D, Drakopoulos M, Vest M, Cooper M, Adderley WP, Wess TJ (2004) Microfocus X-ray diffraction of historical parchment reveals variations in structural features through parchment cross sections. Nano Letters 4:1373-1380

6 Ghioni C, Hiller JC, Kennedy CJ, Aliev AE, Odlyha M, Boulton M, Wess TJ (2005) Evidence of a distinct lipid fraction in historical parchments: a potential role in degradation?. J Lip Res 46:2726-2734

7 Strlič M, Kralj Cigić I, Rabin I, Kolar J, Pihlar B, Cassar M (2009) Autoxidation of lipids in parchment. Polym Degrad Stab 94:886-890.

8 Možir A, Strlič M, Trafela T, Kralj Cigić I, Kolar J, Deselnicu V, de Bruin G (2010) On oxidative degradation of parchment and its non-destructive characterisation and dating. Appl Phys A Doi:10.1007/s00339-0106108-z

9 Popescu C, Budrugeac P, Wortmann FJ, Miu L, Demco DE, Baias M (2008) Assessment of collagen-based materials which are supports of cultural and historical objects. Polym Degrad Stab 93:976-982

10 Gonzalez L, Wess T (2008) Use of attenuated total reflection-Fourier transform infrared spectroscopy to measure collagen degradation in historical parchment. Appl Spectr 62:1108-1114

11 Mannucci E, Pastorelli R, Zerbi G, Bottani CE, Facchini A (2000) Recovery of ancient parchment:characterization by vibrational spectroscopy. J Ram Spectr 31:1089-1097 
12 Edwards HGM, Farwell DW, Newton EM, Rull Perez F, Jorge Villar S (2001) Application of FT-Raman spectroscopy to the characterisation of parchment and vellum, I; novel information for paleographic and historiated manuscript studies. Spectrochim Acta A 57:1223-1234

13 Larsen R (2002) Microanalysis of parchment. Archetype, London

14 Larsen R, Vest M, Nielsen KJ (1993) Determination of hydrothermal stability (shrinkage temperature) of historical leather by the micro hot table technique. J Soc Leath Technol Chem 77:151-156

15 Cohen SN, Odlyha M, Foster GM (2000) Measurement of shrinkage behaviour in leather and parchment by dynamic mechanical thermal analysis. Thermochim Acta 365:111-117

16 Kennedy CJ, Vest M, Cooper M, Wess TJ (2004) Laser cleaning of parchment: structural, thermal and biochemical studies into the effect of wavelength and fluence. Appl Surf Sci 227:151-163

17 Koenig JL (1999) Spectroscopy of polymers. Elsevier, London

18 Jabs $A$ (2011) Determination of secondary structure in proteins by Fourier transform infrared spectroscopy (FTIR), IMB, Jena. http://www.imb-jena.de/ImgLibDoc/ftir/IMAGE_FTIR.htm. Accessed 1 March 2011

19 Derrick M (1991) Evaluation of the state of degradation of Dead Sea scroll samples using FT-IR spectroscopy. The Book and Paper specialty group session, AIC 19th Annual Meeting, June 3-8, Albuquerque, New Mexico

20 Maxwell CA, Wess TJ, Kennedy CJ (2006) X-ray diffraction study into the effects of liming on the structure of collagen. Biomacromolecules 7:2321-2326.

21 Wess TJ, Orgel JP (2000) Changes in collagen structure: drying, dehydrothermal treatment and relation to long term deterioration. Thermochim Acta 365:119-128

22 Hiller JC, Thompson TJU, Evison MP, Chamberlain AT, Wess TJ (2003) Bone mineral change during experimental heating: an X-ray scattering investigation. Biomaterials 24:5091-5097

23 Wess T, Alberts I, Hiller J, Drakopoulos M, Chamberlain AT, Collins M (2001) Microfocus small angle X-ray scattering reveals structural features in archeological bone samples: detection of changes in bone mineral habit and size. Calcif Tissue Int 70:103-110

24 Karmer R (1998) Chemometric techniques for quantitative analysis. Dekker, New York, NY

25 Wiedemann SCC, Hansen WG, Snieder M, Wortel VAL (1998) NIR calibration in practice. Analusis 26:M 38M 43

26 Trafela T, Strlič M, Kolar J, Lichtblau DA, Anders M, Pucko Mencigar D, Pihlar B (2007) Nondestructive analysis and dating of historical paper based on IR spectroscopy and chemometric data evaluation. Anal Chem 79:6319-6323

27 Persikov AV, Ramshaw JAM, Kirkpatrick A, Brodsky B (2000) Amino Acid Propensities for the Collagen Triple-Helix. Biochemistry 39: 14960-14967 